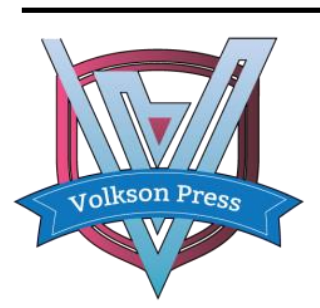

Contents List available at VOLKSON PRESS

New Materials and Intelligent Manufacturing (NMIM)

DOI : http://doi.org/10.26480/icnmim.01.2018.394.397

Journal Homepage: https://topicsonchemeng.org.my/

ISBN: 978-1-948012-12-6

\title{
DESIGN AND ANALYSIS OF A LOW FRICTION TANTALUM POWDER FORMING MOULD UTILIZING MULTI-WAVELENGTH EXCITING MODE
}

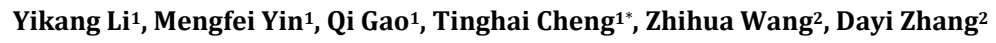 \\ ${ }^{1}$ Department of Mechatronic Engineering, Changchun University of Technology, Yan 'an street, number 2055, Changchun 130012, China \\ ${ }^{2}$ Changchun wei hong dongguang electronic equipment co., LTD, Chuangxin Street, number 215, Changchun 130012, China \\ *Corresponding Author Email: chengtinghai@163.com
}

This is an open access article distributed under the Creative Commons Attribution License, which permits unrestricted use, distribution, and reproduction in any medium, provided the original work is properly cited.

\section{ARTICLE DETAILS}

\section{Article History:}

Received 26 June 2018

Accepted 2 July 2018

Available online 1 August 2018

\section{ABSTRACT}

Tantalum capacitor has been widely used in many fields of electrical, communications, automotive, military, aerospace, etc. This paper proposes a low frictional mould of tantalum capacitor, and the ultrasonic friction reduction is realized by an ultrasonic transducer driven by twelve rectangular piezoelectric plates. The forming pressing mould mainly contains top convex mould, top adjusting shim, ultrasonic transducer, bottom convex mould, and bottom adjusting shim. The rectangular piezoelectric plates are bonded to the opposite outside surfaces of ultrasonic transducer and the d31 working mode is adopted. The composite exciting method of the forming pressing mould is clarified in detail. The modal shape of the ultrasonic transducer is analyzed using Finite Element Method (FEM). The prototype is fabricated and tested. The testing results indicate that the vibration performance of the motor is in good agreement with the simulation results.

\section{KEYWORDS}

Forming mould, Ultrasonic friction reduction, Multi-wavelength exciting mode, Ultrasonic transducer, Finite Element Method (FEM).

\begin{abstract}
1.INTRODUCTION
Tantalum capacitors were first developed in 1956 by the Baer laboratory in United States. It is the product with the excellent performances that a small capacitor volume can achieve a large capacity of capacitance. Tantalum capacitors have various shapes and is easy to make small and chip components suitable for the surface mounting, which meets the development needs of the automation and miniaturization of electronic technology [1,2]. Tantalum capacitors have been widely used in many advanced fields of the electrical, communications, automotive, military, aerospace, etc, because of the characteristics of miniaturization, high capacity, low leakage current, high efficiency, high reliability, long service 1ife [3-6].
\end{abstract}

Tantalum core is one of the important component of tantalum capacitors. The density uniformity of tantalum core obviously affects the performance of the tantalum capacitors. According to the production technology, the fabrication process of tantalum core can mainly be divided into the forming, sintering, energizing, assembly, and the inspection. The forming process is the first technology of the tantalum core production. The forming process has a crucial impact on the density uniformity of tantalum core, and decides the production quality and working performance of tantalum capacitor [7-9].

Researchers across the world have carried out a lot of research work on improving the density uniformity of tantalum core. Huang et al proposed an isostatic pressing mould, which can enable the powder to be subjected to uniform and stable pressure during pressing. The proposed mould is good for pressing the parts of the complex shape [10]. Liu et al designed a high-speed pressing mould, and the traditional static pressure is changed into the dynamic pressure and the density of powder compact is improved. However, the production cost of high-speed pressing mould is high, and the mould loss is seriously [11]. Hoeganaes company proposed the warm compaction forming mould, and the influence of the temperature and lubrication on warm compaction technology has been extensively studied. However, the warm compaction technology is complex and requires high pretreatment of the powder [12, 13]. In addition, Ye et al applied the powder lubrication technology to the traditional pressing mould. Although this method plays a positive role in improving powder density, it is difficult to completely remove from the powder mould due to the harsh lubricants environment [14]. Thus, it is much necessary to proposed a novel principle of the tantalum forming pressing process, which has met the technical requirements of the advanced fields.

As an effective method for decreasing friction force, the ultrasonic friction reduction has been widely employed in materials production, the density uniformity of tantalum core can be improved by the friction force between tantalum powder particles and mould wall [15-18]. In this paper, a low frictional tantalum powder forming mould based on ultrasonic friction reduction is proposed to improve the density uniformity of tantalum core, which can be achieved by the three-wavelength exciting mode. The exciting mode of forming pressing mould is analyzed and its vibration performance is simulated by the Finite Element Method (FEM). A prototype of the forming mould is fabricated and the vibration characteristics are also tested.

\section{STRUCTURE OF THE TANTALUM POWDER FORMING MOULD}

Figure 1. shows the structure schematic diagram of tantalum powder forming mould, and it is mainly consisted of a top convex mould, a top adjusting shim, a dodecagon concave, twelve rectangular piezoelectric plates, a bottom convex mould, and a bottom adjusting shim, as shown in figure 1(a). The designed forming mould comprised of a ultrasonic transducer that is a dodecagon concave mould bonded with twelve rectangular piezoelectric plates on its external surface at 30 degree 
spacing. The outer diameter of the dodecagon concave mould is $\$ 40 \mathrm{~mm}$, and its length is $56 \mathrm{~mm}$. The dodecagon concave mould is set up four blind holes, such as holes $B_{1}, B_{2}, B_{3}$ and $B_{4}$, and the diameter of the holes $B_{1}, B_{2}$, and $B_{3}$ is $9 \mathrm{~mm}$, and its depth is $50 \mathrm{~mm}$. The diameter of hole $B_{4}$ is $8 \mathrm{~mm}$, and its depth is $48 \mathrm{~mm}$. The stiffness and exciting frequency of the forming mould can be adjusted by changing the diameters and depth of holes $B_{1}$, $\mathrm{B}_{2}, \mathrm{~B}_{3}$ and $\mathrm{B}_{4}$.

The dodecagon concave mould is also set up three through holes, such as holes $\mathrm{C}_{1}, \mathrm{C}_{2}$, and $\mathrm{C}_{3}$. The length, width, and thickness dimensions of the piezoelectric plate are $16 \mathrm{~mm}, 6 \mathrm{~mm}$, and $1 \mathrm{~mm}$, respectively. The top and bottom adjusting shims are used to adjusting the gap between convex moulds and dodecagon concave mould. The top convex mould is set up with three through holes, such as the holes $A_{1}, A_{2}$, and $A_{3}$, and the diameter of the through holes are about $0.6 \mathrm{~mm}$, which are mainly used for the placement of tantalum wire. The designed forming mould has the advantages of simple structure. Therefore, the lower cost and the batch fabrication for the tantalum forming mould can be easily achieved.

(a)

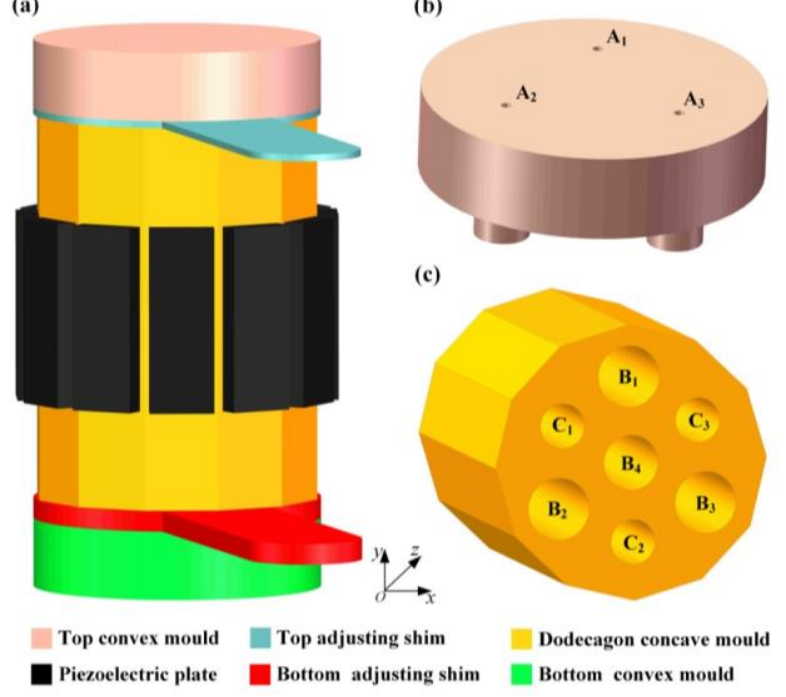

Figure 1: Structure of the forming pressing mould

\section{THE EXCITING METHOD OF ULTRASONIC TRANSDUCER}

The exciting method of the proposed tantalum powder forming mould based on ultrasonic friction reduction is shown in figure 2. The ultrasonic friction reduction effect of ultrasonic transducer is achieved by multiwavelength exciting mode. The larger vibration amplitude of the ultrasonic transducer can be obtained, and the better ultrasonic friction reduction effect is achieved. This exciting method can be achieved by the multi-wavelength travelling wave that can be synthesized by the third and first bending vibration modes along the circumferential and axial directions, respectively. In order to excite the forming mould to produce the multi-wavelength travelling wave processing along the circumferential direction, the twelve rectangular piezoelectric plates are polarized in the direction of thickness and the $d_{31}$ working mode is adopted twelve rectangular piezoelectric plates, respectively. The six rectangular piezoelectric plates, from the $\mathrm{M}_{1}$ to $\mathrm{M}_{6}$, constitute the exciting group M.
The exciting group $\mathrm{N}$ includes the rest of six rectangular piezoelectric plates, from the $N_{1}$ to $N_{6}$. Simultaneously, the concave mould with the internal surface is connected with the tantalum powder particles. The standing wave vibrations $S_{M}$ and $S_{N}$ with three crests and troughs can be generated by the exciting signals $U_{0} \sin \omega t$ and $U_{0} \sin (\omega t+\varphi)$, respectively. The excitation frequency of the exciting groups $\mathrm{M}$ and $\mathrm{N}$ is supposed to be coincident with the resonant frequency of the dodecagon concave mould. When the phase shift between the exciting groups $\mathrm{M}$ and $\mathrm{N}$ is $90^{\circ}$, a threewavelength travelling wave can be synthesized by the standing wave vibrations $S_{M}$ and $S_{N}$. The vibration amplitude can be effectively magnified by the three-wavelength travelling wave, because the effective contact area between concave mould with the internal surface and tantalum powder particles is increased. Thus, the small friction force can be obtained, and the density uniformity of the tantalum core is effectively improved. When the phase shift between the exciting groups $\mathrm{M}$ and $\mathrm{N}$ is $270^{\circ}$, the direction of the vibration amplitude can be adjusted. The small friction force are still obtained, and the same output performance can be achieved relative to the phase shift of $90^{\circ}$. Here, the "arrow" represents the polarized direction of the rectangular piezoelectric plates.

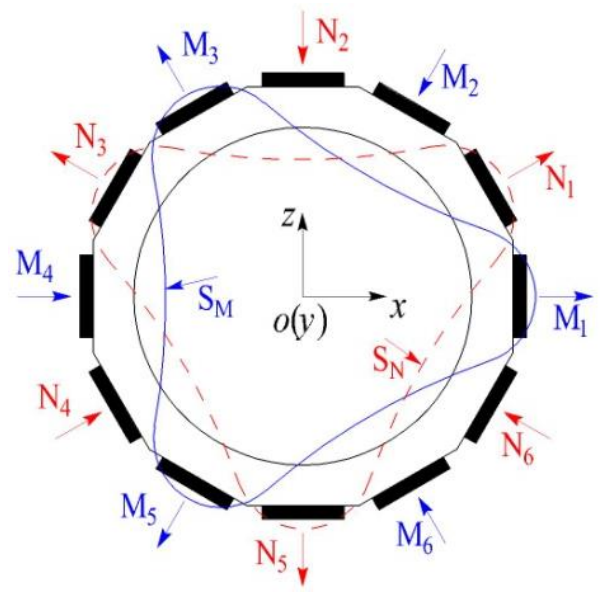

Figure 2: The composite exciting method of the forming pressing mould

\section{SIMULATION ANALYSIS OF ULTRASONIC TRANSDUCER}

The structure of ultrasonic transducer includes the twelve rectangular piezoelectric plates and dodecagon concave mould. The free beam constraint is applied to ultrasonic transducer. The vibration characteristics of ultrasonic transducer are analyzed using the Finite Element Method (FEM). During the simulation analysis, some important parameters are set as follows: the Young's modulus $E$, Poisson ratio $\sigma$, and Mass density $\rho$ of the dodecagon concave mould are $218 \mathrm{GPa}, 0.28,7850$ $\mathrm{kg} / \mathrm{m}^{3}$, respectively. The piezoelectric plates are polarized in the direction of thickness and $d_{31}$ working mode is adopted respectively. The type of PZT4-2 is adopted for rectangular piezoelectric plates (Bao Ding Hong Sheng Acoustics Electron Apparatus Co., Ltd). The density of the twelve rectangular piezoelectric plates is $7500 \mathrm{~kg} / \mathrm{m}^{3}$. The other mechanical and physical parameters of the rectangular piezoelectric plates are listed in Table 1.

Table 1: Mechanical and physical parameters of rectangular piezoelectric plates

\begin{tabular}{|cc}
\hline Dielectric coefficient matrix $(\mathrm{F} / \mathrm{m})$ & Piezoelectric stress matrix $\left(\mathrm{C} / \mathrm{m}^{2}\right)$ \\
\hline$[\varepsilon]=\left[\begin{array}{ccc}7.30 & 0 & 0 \\
0 & 7.30 & 0 \\
0 & 0 & 6.35\end{array}\right] \times 10^{-9}$ \\
{$[\mathrm{GPa})$}
\end{tabular}


A finite element model of ultrasonic transducer was established in ANSYS Workbench software, as shown in figure 3. The modal analysis was carried out. The Free Meshing and Mapped Meshing were adopted for dodecagon concave mould and rectangular piezoelectric plates, respectively. The glue layers were neglected because they are too thin to be meshed and the deviations can be acceptable. The simulation result indicates that the combined circumferential-axial mode of the ultrasonic transducer can be excited and resonant frequency of ultrasonic transducer is about 53.11 $\mathrm{kHz}$. The simulation result provides an important reference for the selection of the resonant frequency in the multi-wavelength exciting mode.

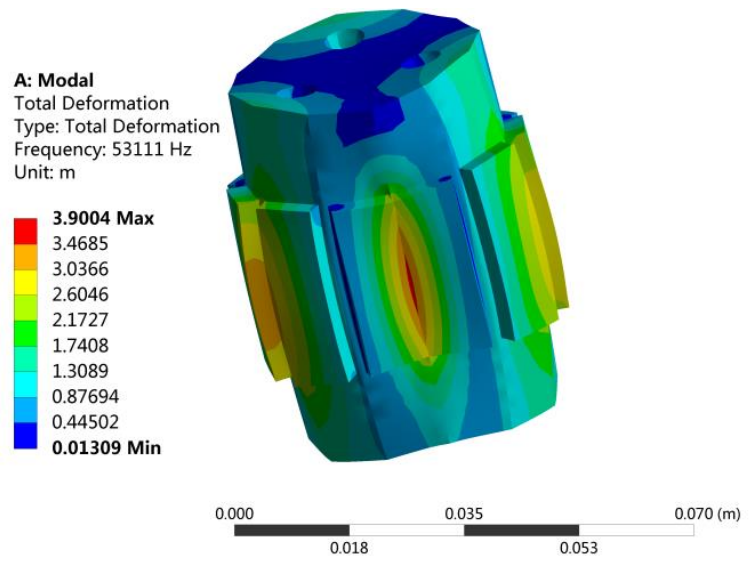

Figure 3: Working modal shape of the ultrasonic transducer by FEM

\section{FABRICATION AND TEST OF PROTOTYPE}

According to the structure design and the simulation analysis, a prototype of the low frictional tantalum powder forming mould utilizing the multiwavelength exciting mode is fabricated and its photograph is shown in figure 4 . The designed prototype mainly includes the top convex mould, the top adjusting shim, the dodecagon concave, twelve rectangular piezoelectric plates, the bottom convex mould, and the bottom adjusting shim. The $\mathrm{Cr} 12 \mathrm{MoV}$ is chosen as the material for the top convex mould, dodecagon concave, and bottom convex mould because of its high stiffness and low density. The stainless steel is chosen as the material for the top adjusting shim and bottom adjusting shim. The tantalum core is formed by the sliding contact between the through holes $\mathrm{C}_{1} \sim \mathrm{C}_{3}$ and the top convex mould and the bottom convex mould. In the process of the tantalum powder forming pressing, the pressing becomes tighter with the decreasing adjusting shim. Under the excitation of voltage, the ultrasonic transducer can generate the micro ultrasonic vibration with high frequency and reduce the friction between the tantalum powder and the wall of dodecagon concave mould. The density uniformity of tantalum core can be improved.

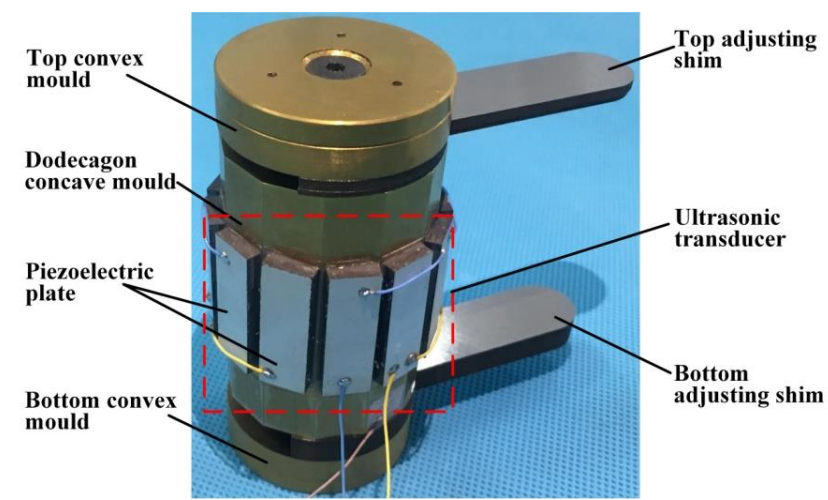

Figure 4: Prototype photograph of tantalum powder forming mould

During the experiments, an impedance analyzer (PV70A) was used to test the vibration performance of the designed prototype. Figure 5 illustrates the impedance testing results under the scanning frequencies from 54.00
$\mathrm{kHz}$ to $55.40 \mathrm{kHz}$. The impedance characteristics testing result indicates that the resonant frequency of the prototype is $54.69 \mathrm{kHz}$. There has a frequency deviation of $1.58 \mathrm{kHz}$ between the testing results and the simulation analysis results. The major reasons of the deviation are the actual and the simulation material parameters, manufacturing and assembly errors, and so on

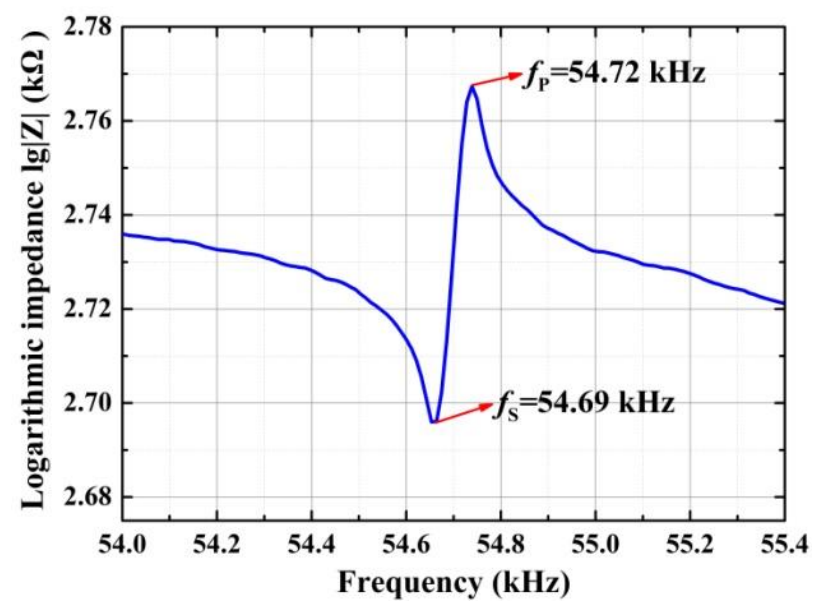

Figure 5: Vibration performance of the prototype

\section{CONCLUSION}

A low frictional tantalum powder forming mould utilizing the multiwavelength exciting mode was proposed in this paper. The designed forming mould was composed of a top convex mould, a top adjusting shim, a ultrasonic transducer, a bottom convex mould, and a bottom adjusting shim. The driving travelling wave along the circumferential direction of the ultrasonic transducer was composited by the orthogonal standing wave vibrations, and the ultrasonic friction reduction is realized by an ultrasonic transducer. The friction force of the ultrasonic transducer with inner surface is effectively decreased based on ultrasonic friction reduction. The Finite Element Method (FEM) was adopted to analyze the working modal shape of the ultrasonic transducer. A prototype of the motor was fabricated and it's vibration characteristic was tested. The testing results are well consistent with the simulation analysis results. Our future work will be focused on the establishment of testing system and study on pressing characteristics of the tantalum powder forming mould.

\section{ACKNOWLEDGMENTS}

This work was financially supported by the Technology Development Plan of Jilin Province (No. 20160204054GX), and the National Natural Science Fund of China (No. 51775130), and the Key Projects of Science and, Technology Research Planning Project of Education Department of Jilin Province (No. JJKH20181037KJ).

\section{REFERENCES}

[1] He, J.L. 2001. The development of world tantalum powder technology. Engineering Science, 3 (12), 85-89. doi: 10.3969/j.issn.10091742.2001.12.015

[2] He, J.L. 2004. New development of tantalum and niobium electronic materials. The Chinese Journal of Nonferrous Metals, 23 (2), 291-300. doi: 10.3321/j.issn:1004-0609.2004.z1.046

[3] Obuszewski, M. 2003. Multilayer ceramics or tantalums. Power Electronics Technology, 29 (5), 16-18.

[4] Liu, S.Y. 1998. Applications of tantalum in High-tech. Rare Metals and Cemented Carbides, (2), 55-57.

[5] Orlov, V.M., Kolosov, V.N., Prokhorova, T.Y. 2007. Production of Tantalum Capacitor Powders with a Large Specific Surface Area. Theoretical Foundations of Chemical Engineering, 41 (5), 585-588. doi: $10.1134 /$ S0040579507050211

[6] Baba, M., Suzuki, R.O. 2005. Dielectric properties of tantalum powder with broccoli-like morphology. Journal of Alloys and Compounds, 392 (1), 225-230. doi: 10.1016/j.jallcom.2004.09.039 
[7] Liu, Y.G., Yi, J.F. 2008. The influence of forming Sintering process on the electrical properties of tantalum capacitors with solid electrolyte. Rare Metals and Cemented Carbides, 36, 17-20.

[8] Yoon, J.S., Kim, B.I. 2007. Characteristics and production of tantalum powders for solid-electrolyte capacitor. Journal of Powder Sources, 164 (2), 959-963. doi: 10.1016/j.jpowsour.2006.11.035

[9] Fujihara, Y., Watanabe, H., Takino, K. 2009. A method for the preparation of the raw powder of the tantalum solid electrolytic capacitor. Journal of the Japan Society of Powder and Powder Metallurgy, 44 (8), 746-750. doi: $10.2497 /$ jjspm.44.746

[10] Huang, P.Y. 1997. Theory of powder metallurgy. 2nd ed. Beijing: Metallurgical Industry Press, 218.

[11] Guo, S.J., Chi, Y., Meng, F. 2006. Compaction equation for high velocity compact shaping of powder metallurgy. Materials Science and Engineering of Powder Metallurgy, 11, 24-27.

[12] Cao, S.H., Lin, X.P, Li, J.Y. 2006. Compaction technology of iron-based powder mixtures with heating die. Materials Science and Engineering of Powder Metallurgy, 11 (4), 201-205.

[13] Bocchini, G.F. 1999. Warm compaction of metal powders: Why it works, why it requires a sophisticated engineering approach. Powder Metallurgy, 42, 171-180.

[14] Ye, T.M., Yi. J.H., Chen S.J. 2005. Die wall lubricated warm compaction behavior of non-lubricant admixed iron powders. Journal of Central South University, 12 (6), 653-656. doi: 10.1007/s11771-005-0063-4

[15] Thoe, T.B., Aspinwall, D.K., Wise, M.L.H. 1988. Review on ultrasonic machining. International Journal of Mechanical Tools Mantifacture, 38 (4), 239-255. doi: 10.1016/S0890-6955(97)00036-9

[16] Tsujino, J., Yajbuzaki, M., Ueoka, T. 1987. Studies on ultrasonic vibration press of powder by longitudinal vibration punches and various vibration mode dies. Japanese Journal of Applied Physics, 26 (S1), 209211.doi: 10.7567/JJAPS.26S1.209

[17] Ghaffar, F., Badshah, N., Islam, S. 2017. Multigrid Solution for The Cauchy Problem Associated with Hel mholtz Type Equation on NonUniform Grids. Matrix Science Mathematic, 1 (2), 06-10.

[18] Tsujino, J., Saotome, Y., Shimada, N. 2009. Compacting of various metal, alloy and ceramic powder using $20 \mathrm{kHz}$ ultrasonic vibration compacting equipments with upper and lower vibration punches. IEEE International Ultrasonics Symposium Proceedings, 1985-1988. doi: 10.1109/ULTSYM.2009.5441788.

\section{ABOUT THE AUTHORS}

Yikang Li was born in Jilin province, China, in 1994. He received the B.S. degree from the Changchun University of Technology, Changchun, China, in 2017. He is currently pursuing the M.S. degree with the Department of Mechanical Engineering, Changchun University of Technology. His research interests include piezoelectric actuators and smart materials and structures.

Mengfei Yin was born in Shangdong province, China, in 1994. He received the B.S. degree from the Changchun University of Technology, Changchun, China, in 2017. Now, he is studying for a master's(M.S.) degree in mechanical engineering at Changchun University of Technology. His research interests include friction drive mechanism and piezoelectric energy harvesting.

Qi Gao was born in 1995 Jilin province, China, majored in mechanical engineering and achieved the B.E. degree from Changchun University of Technology in 2017. He continues pursuing a Master of Engineering degree in the same School. His current main research direction is piezoelectric actuators and precision machinery.

Tinghai Cheng was born in Heilongjiang province, China. He received the B.S. degree in mechanical design manufacturing and automation, M.S. degree in mechanical design and theory, and Ph.D. degree in the mechatronic engineering from the Harbin Institute of Technology, Harbin, China, in 2006, 2008, and 2013, respectively. Currently, he is an Associate Professor with the Department of Mechatronic Engineering, Changchun University of Technology, Changchun, China. His current research interests include piezoelectric actuators and piezoelectric energy harvesting

Zhihua Wnag was born in Jilin province, China, in 1977. He received the B.S. degree from the Chengdu University of Technology, Chengdu, China, in 2000 . He is currently working in the Changchun wei hong dongguang electronic equipment co., LTD. His research interests include electronic components manufacturing and management.

Dayi Zhang was born in Jilin province, China, majored in molecular material processing machinery and achieved the B.E. degree from Beijing University of Chemical Technology in 1997. Now, he is working in the Changchun wei hong dongguang electronic equipment co., LTD. His research interests include electronic components. 